Methods We conducted 27 semi-structured interviews with purposeful sample of researchers who have developed, implemented or evaluated SBSHI in sSA. Interviews were audio recorded, transcribed verbatim and analysed thematically. Intervention Mapping framework (IM) was used to organise emerging themes.

Results Participants are experts in SBSHI in sSA with experiences ranging between 2-30 years. We identified 33 themes that mapped onto the six steps of IM. During development, social factors such as poverty and school infrastructures including quality of teaching, in addition to sexual health needs, should be addressed. SBSHI should be culturally sensitive, address inter-generational and open communications on sexual issues, and clearly state aims to avoid any misunderstanding. Curriculum should also address contemporary issues in HIV prevention (Treatment as Prevention, Pre-exposure Prophylaxis, Voluntary Medical Male Circumcision and Gender-Based Violence). During implementation, SBSHI should be prepared for oppositions at various levels for which we have also identified effective approaches for overcoming them. Due to limited teachers' training in SSA, provisions of simple but detailed facilitators' manual together with supportive supervisions may be critical in ensuring delivery fidelity. During evaluation, computerised audio devices and qualitative interviews with participant observations may facilitate collection and improve validity of adolescents' sexual behaviours data respectively.

Conclusion Using insights from experiences of researchers, recommendations for developers, implementers or evaluators of SBSHI in sSA that will complement available guidance are provided.

\section{P4.04 EDUCATING HEALTH WORKERS IS KEY IN CONGENITAL SYPHILIS ELIMINATION IN COLOMBIA}

${ }^{1} J u a n$ P Garcés, 'Luisa C Rubiano, 'Yenifer Orobio, ${ }^{2}$ Martha C Castaño, ${ }^{3}$ Elizabeth Benavidez, 'Adriana R Cruz. Centro Internacional de Entrenamiento e Investigaciones Médicas (CIDEIM), Cali, Colombia; 2 Secretaría de Salud Departamental del Valle del Cauca. Cali, Colombia; 3 Secretaría de Salud Pública Municipal de Santiago de Cali. Cali, Colombia

\subsection{6/sextrans-2017-053264.50}

Introduction Colombia promotes the diagnosis and treatment of gestational syphilis in a single visit using rapid diagnostic tests to prevent mother-to-child transmission. Additionally, integrated health programs pursue the coordinated prevention of mother-to-child transmission of syphilis/HIV. Objective: To identify knowledge gaps among health workers in the prevention of mother-to-child transmission of syphilis/HIV and to provide recommendations to support these programs.

Methods We conducted a descriptive study based on 306 surveys of health workers in 39 health institutions in the city of Cali. Surveys inquired about planning-management-implementation of services for pregnant women, clinical knowledge of $\mathrm{HIV} /$ syphilis/rapid diagnostic tests, and prior training.

Results Knowledge deficits in the management of gestational syphilis were detected among the surveyed health workers, including physicians. Rapid tests for syphilis are currently used in clinical laboratories in Cali, however, procedural deficiencies were observed in their use, including quality control assurance. During the two years prior to the survey, training of health workers in the prevention of mother-to-child transmission of
syphilis/HIV has been limited. Health workers are interested in identifying and treating gestational syphilis in a single event, in using rapid diagnostic tests and in receiving training. Conclusion Intensive training that targets health workers, policy/decision makers and academic groups is needed to ensure adequate implementation of new strategies for the prevention of mother-to-child transmission of syphilis/HIV.

\section{P4.05 MIGRATION AND ACCULTURATION: UNDERSTANDING TRANSGENDER NETWORK SOCIALISATION PATTERNS IN LIMA, PERU}

${ }^{1}$ Amaya Perez-Brumer, ${ }^{2}$ Ximena Salazar, ${ }^{3}$ Sarah Mclean, ${ }^{2}$ Alfonso Silva-Santisteban, ${ }^{4}$ Javier R Lama, ${ }^{4} J$ orge Sanchez, ${ }^{5}$ Matthew J Mimiaga, ${ }^{6}$ Sari L Reisner, ${ }^{3}$ Ken H Mayer, ${ }^{7}$ Jesse L Clark. 'Columbia University Mailman School of Public Health, New York, USA; ${ }^{2}$ Universidad Peruana Cayetano Heredia, Lima - Peru; ${ }^{3}$ The Fenway Institute, Fenway Health, Boston, USA; ${ }^{4}$ Asociación Civil Impacta Salud Y Educación, Lima - Peru; ${ }^{5}$ The Fenway Institute, Fenway Health; Department of Behavioural and Social Sciences, Brown University, Providence, USA; ${ }^{6}$ The Fenway Institute, Fenway Health; Paediatrics, Boston Childrens Hospital and Harvard Medical School, Boston, USA; 'Department of Medicine, Division of Infectious Diseases, University of California, Los Angeles, Los Angeles, USA

\subsection{6/sextrans-2017-053264.502}

Introduction Community acceptability and sustainability of new HIV prevention methods remain key barriers to curb high HIV incidence rates for transgender women (TW) in Peru. Improved understanding of how geography, culture, and socialisation influence $\mathrm{HIV}$-associated risks and uptake of new prevention technologies is critical to address the HIV epidemic among TW.

Methods In 2015-2016, 20 interviews and demographic surveys were conducted with TW in Peru to inform a social network-based PrEP adherence intervention. (Interviews probed on: socialisation, social network formations, preferred HIV intervention methods). Audio files were transcribed verbatim and analysed via immersion crystallisation using Dedoose software.

Results TW had a mean age of 30 years, $40 \%(n=8)$ reported sex work as their primary occupation, and 60\% $(n=12)$ had migrated from another part of Peru to Lima. Principal motivations for migration were: lack of economic opportunity, familial rejection due to gender identity, and seeking larger communities of TW as found in Lima. Co-habitation with other TW was common, underscoring cluster structures of socialisation, acculturation to existing norms (i.e., learning the rules of sex work in Lima), and knowledge-sharing. Among TW migrants, social network clusters were patterned not only on gender identity and occupation, but also province of origin (i.e., Iquitos, Trujillo) through which new arrivals would learn social/cultural norms from established TW community leaders. In addition to shared cultural practices, participants described greater trust and social cohesion with TW from similar geographic areas.

Conclusion Geographic migration highlights the weight of cultural context in informing network structures and defining influences and leadership in TW communities. Efforts to improve TW engagement in HIV prevention will need to better understand how existing social networks influence HIV risk and prevention and how these can be used to address distinct needs of the heterogeneous Peruvian TW population. 